\title{
Velocity Calculation by Automatic Camera Calibration Based on Homogenous Fog Weather Condition
}

\author{
Hong-Jun Song ${ }^{1} \quad$ Yang-Zhou Chen ${ }^{1} \quad$ Yuan-Yuan Gao ${ }^{2}$ \\ ${ }^{1}$ Center for Autonomous Technology and Intelligent Control, Beijing University of Technology, Beijing 100124, China \\ ${ }^{2}$ College of Information and Engineering, Zhejiang Agriculture and Forestry University, Hangzhou 311300, China
}

\begin{abstract}
A novel algorithm for vehicle average velocity detection through automatic and dynamic camera calibration based on dark channel in homogenous fog weather condition is presented in this paper. Camera fixed in the middle of the road should be calibrated in homogenous fog weather condition, and can be used in any weather condition. Unlike other researches in velocity calculation area, our traffic model only includes road plane and vehicles in motion. Painted lines in scene image are neglected because sometimes there are no traffic lanes, especially in un-structured traffic scene. Once calibrated, scene distance will be got and can be used to calculate vehicles average velocity. Three major steps are included in our algorithm. Firstly, current video frame is recognized to discriminate current weather condition based on area search method (ASM). If it is homogenous fog, average pixel value from top to bottom in the selected area will change in the form of edge spread function (ESF). Secondly, traffic road surface plane will be found by generating activity map created by calculating the expected value of the absolute intensity difference between two adjacent frames. Finally, scene transmission image is got by dark channel prior theory, camera's intrinsic and extrinsic parameters are calculated based on the parameter calibration formula deduced from monocular model and scene transmission image. In this step, several key points with particular transmission value for generating necessary calculation equations on road surface are selected to calibrate the camera. Vehicles' pixel coordinates are transformed to camera coordinates. Distance between vehicles and the camera will be calculated, and then average velocity for each vehicle is got. At the end of this paper, calibration results and vehicles velocity data for nine vehicles in different weather conditions are given. Comparison with other algorithms verifies the effectiveness of our algorithm.
\end{abstract}

Keywords: Vehicle velocity calculation, homogenous fog weather condition, dark channel prior, monocular, camera calibration.

\section{Introduction}

In the last decade, much more research has been carried out in the field of intelligent transportation system (ITS) in order to increase the road safety and comfort when driving ${ }^{[1]}$. Consequently, there is an increasing requirement to develop intelligent traffic surveillance systems that can play an important role in highway monitoring and road management systems. Their purpose is to provide real-time statistical data on traffic management and to detect potentially anomalous situations, e.g., dangerous driving. Accurate measurement of the vehicle's speed from road surveillance cameras is a desired goal for more than a decade. Concerning vehicles automatic detection and velocity computation, processing can be based on three major types of methodologies: the use of physical sensing devices (laser, radar and buried coil, etc.), the use of binocular vision, and monocular vision.

The use of physical sensing devices to detect vehicle velocity has been widely spread into the different kinds of industries. But somehow, the equipment itself has some disadvantages. Majority of physical sensing devices dedicated to measure vehicle velocity are expensive to operate and quite often complicated to install correctly. Velocity detection by using radar technology has some disadvantages, such as the cost of equipment, which is the most impor-

Manuscript received April 30, 2012; revised October 31, 2012

This work was supported by National High Technology Research and Development Program of China (863 Program) (No. 2011AA110301), National Natural Science Foundation of China (No.61079001), and the Ph. D. Programs Foundation of Ministry of Education of China (No. 20111103110017). tant reason to find other compensating equipment that can reduce the cost of investment. Buried coil method could not get accurate value and may damage the road. Ki and Baik $^{[2]}$ used double-loop detectors hardware to measure vehicles velocity. However, only local vehicle velocity in a particular location could be detected.

A single camera is only being used in this approach, in common scene, whereas image depth and vehicle distance are unable to gain. This problem has been overcome by adopting the hypothesis of a flat road, which makes it possible to associate a distance with each line of the image ${ }^{[3]}$. Lin and $\mathrm{Li}^{[4]}$ proposed a novel approach for vehicle velocity detection based on motion blurred images. The motion blur parameters are first estimated from the acquired images and then used to detect the velocity of the moving object in the scene. They established a link between the motion blur information of a $2 \mathrm{D}$ image and the velocity information of a moving object. This method is very explicit, however not all the moving objects could leave blurred portrait. Dailey et al. ${ }^{[5]}$ presented a novel algorithm using a sequence of video images from an un-calibrated camera. The algorithm uses frame difference to isolate moving edges and track vehicles between frames. The algorithm uses a prior known vehicle length distribution with image information to estimate velocity.

Camera can detect velocity directly after calibrated. Camera calibration is a method of determining the internal geometry, optical characteristics (intrinsic parameters), the three-dimensional position, and orientation of a camera relative to a certain world coordinate system (extrinsic parameters). Its purpose is to infer the three dimensional 
information from computer image coordinate or deduce computer image coordinate from the three dimensional information ${ }^{[6]}$. In recent two decades, camera calibration has been studied and developed in many universities and scientific institutes. This research has mainly three stages $^{[7]}$ :

1) Traditional camera calibration makes use of the known scene information and commonly adopts a kind of accurate template similar to calibration block. This method is applicable to all camera models and the accuracy of calibration is very high. However, the process of calibration is very complicated, and some structure information must be provided. So, this accurate template cannot be used widely in many practical applications.

2) Active vision calibration mainly uses some movement information in camera video image. This method provides the linear solution and high robustness, but is not applied to the situation of camera movement or uncontrolled camera.

3) The character of self calibration method only depends on corresponding mutual relation among the analytical images. This method has strong flexibility and wider application prospect, but non-linear computing and robustness need to be improved further.

Previous work has also attempted to use either scene information $^{[8]}$ or camera motion ${ }^{[9]}$ to accomplish this calibration $^{[10]}$. The described computer vision techniques require that many specific features be acquired and reidentified over the entire set of images, and these features are distributed over the surface of the scene. This requirement makes camera calibration suitable for scenes with a small depth of field, but ill-suited for the very large depth of field found in typical traffic management camera scenes ${ }^{[10]}$. Todd $^{[10]}$ presented a new three-stage algorithm to calibrate roadside traffic management cameras and track vehicles to create a traffic velocity sensor. The algorithm first estimates the camera position relative to the roadway using the motion and edges of the vehicles. Then, the algorithm calibrates the camera by estimating the lane boundaries and the vanishing point of the lines along the roadway. The algorithm transforms the image coordinates from the vehicle tracker into real-world coordinates using the simplified camera model. Instead of using painted lines in the image with the required geometry, Todd used the traffic flow to establish an activity map (corrected for nonzero vehicle height) and the hough transform to identify the parallel roadway edges.

For binocular vision algorithms, three steps are needed for traditional binocular system. Firstly, the internal and external parameters for both cameras should be regulated and the relationship for these two cameras should be ascertained. Secondly, matching for each pixel will be done between the left image and the right image. Finally, scene depth is calculated based on the distance computation formula. By using binocular vision, depth and distance for the whole scene could be got and then vehicle velocity could be calculated. However, a number of major problems exist in the $3 \mathrm{D}$ velocity estimation system. One of the most important problems is the running time (mainly in the matching stage). It is so long that could not maintain the real time processing. More importantly, most pixels between left and right traffic images are so similar that they are difficult to be distinguished because of their union color and texture.

All the algorithms listed above neglect the implicit distance information which exists in light transmission model in air especially when it is homogenous fog weather condition. This light propagation model connects scene distance, extinction coefficient and transmission with each other. We may know camera parameters which could be used to compute camera coordinate and vehicle velocity directly as long as we know certain relationship between the extinction coefficient and traffic scene transmission. He et al. ${ }^{[11]}$ proposed a method of estimating scene transmission using dark channel prior. This dark channel prior is based on the following observation on fog-free outdoor images: in most of the nonsky patches, at least one color channel has very low intensity at some pixels. In other words, the minimum intensity in such a patch should have a very low value. Camera parameter calibration should be implemented in homogenous fog weather condition, and it could be used in any weather condition.

A novel algorithm for vehicle average velocity detection through automatic and dynamic camera calibration based on dark channel prior in homogenous fog weather condition is presented in this paper. Unlike past researches in velocity detection area, our traffic model only includes road plane and vehicles in motion. Painted lines in scene image are neglected because sometimes there are no traffic lanes, especially in un-structured traffic scene. Once calibrated, scene distance will be got and can be used to calculate vehicles average velocity. Three major steps are included in our algorithm. Firstly, current weather condition is recognized to justify whether it is homogenous weather condition or not based on area search method (ASM). If it is homogenous fog, average pixel value from top to bottom in the selected area will change in the form of edge spread function (ESF). Secondly, traffic road surface plane will be found by generating activity map created by calculating the expected value of the absolute intensity difference between two adjacent frames. Finally, scene transmission image is got by dark channel prior theory, camera's intrinsic and extrinsic parameters are calculated based on the parameter calibration formula deduced from monocular model and scene transmission image. In this step, several key points with particular transmission value for generating necessary calculation equations on road surface are selected to calibrate the camera. Vehicles' pixel coordinates are transformed to camera coordinates. Distance between vehicles and the camera will be calculated, and then average velocity for each vehicle is got.

This article is organized as follows. Section 2 gives an overview and lists the hypothesis of the whole article. Section 3 indicates homogenous fog weather condition recognition algorithm and corresponding recognition results. Section 4 introduces the monocular model, particular model for traffic plane, coordinates transformation formula, dark channel prior theory, road surface plane extraction method, ultimate camera parameter calculation formula, and points selection principle for generating calculation formula. Section 5 introduces the distance calculation formula based on camera calibration and velocity calculation formula. Section 6 gives the parameter calibration and nine vehicles velocity detection results. Parameter calibration static data 
compared with the other algorithms verify our proposed algorithm. Finally, a conclusion ends this paper.

\section{Algorithm flow and underlying as- sumptions}

\subsection{Several assumptions for this proposed algorithm}

We indicate some facts and make several assumptions to simplify the problem to estimate velocity from traffic video images:

1) Velocity of the vehicles is finite. The velocity of a vehicle has both physical and legal limits.

2) Vehicles' movement is smooth. There are no sudden changes of direction in the time interval $(330 \mathrm{~ms})$ between frames in the image sequence.

3) Homogenous fog weather condition is a common weather phenomenon. According to the China meteorological official statistics, it can almost account up to $40 \%$ of all the weather conditions.
4) Motion is constrained to the road plane. Tracking of vehicles in the image sequence is a one dimensional problem.

5) Camera height (the only prior parameter) is assumed to be $6 \mathrm{~m}$.

\subsection{Overall framework for our algorithm}

Our system mainly includes three different modules: weather condition recognition module, camera calibration module, and vehicle velocity calculation module. Weather condition recognition module includes four different parts: model selection, texture detection, region selection, and edge spread function (ESF) for judging. Camera calibration module includes four different parts: scene transmission calculation based on dark channel prior, road surface extraction, key points selection for formula generation, and parameter calibration. Finally, the vehicle velocity calculation module includes three parts: vehicle tracing, vehicle moving distance calculation, and velocity calculation. Fig. 1 shows this proposed algorithm processing framework.

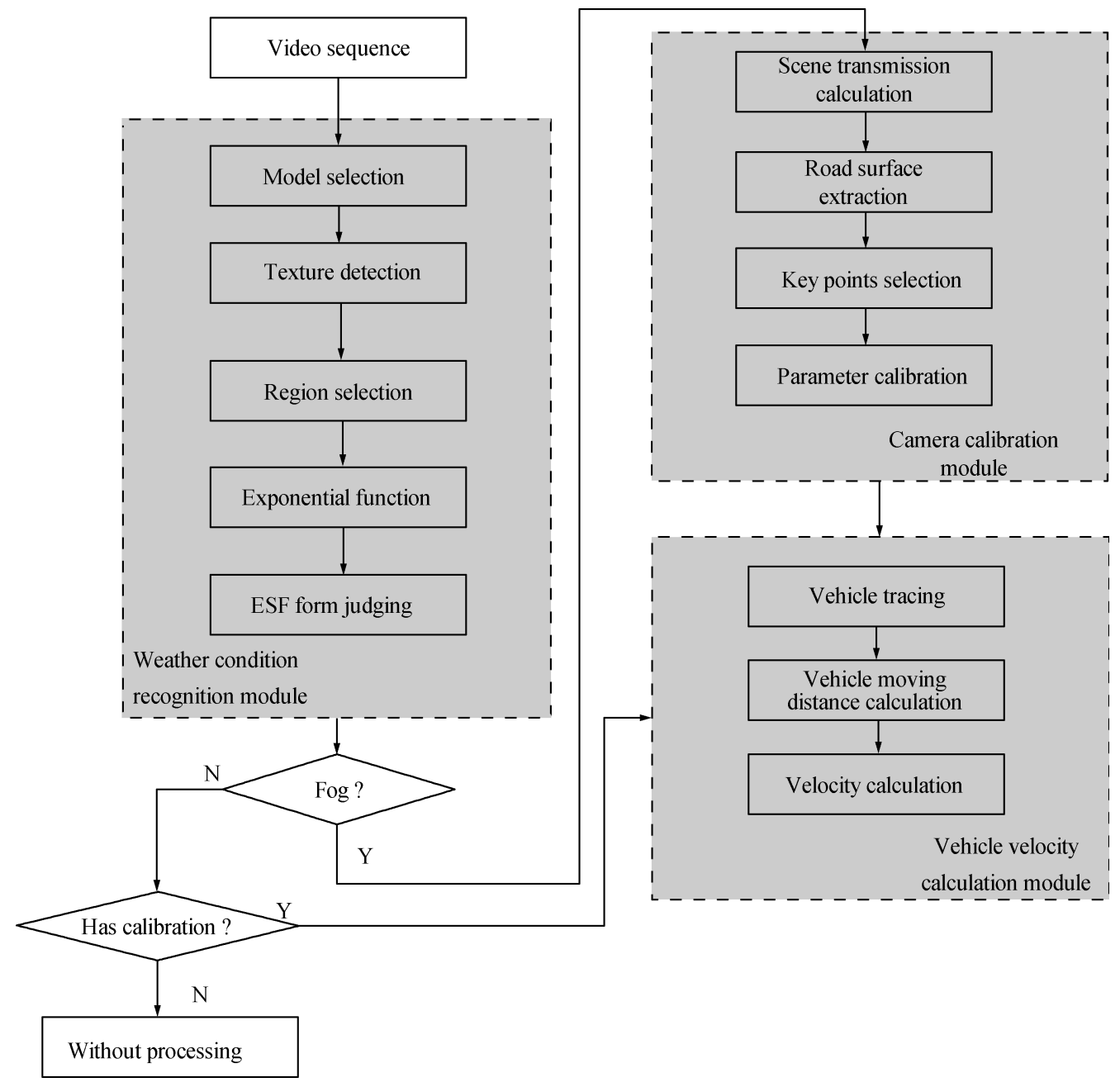

Fig. 1 Algorithm flowchart 


\section{Homogenous fog weather condition recognition}

\subsection{Light transmission model in fog weather condition}

Light traveling through a scattering medium is attenuated along its original course and is distributed to other directions. This process is commonly modeled mathematically by assuming that there is a linear relation between the fractions of the deflected light and the traveled distance along short distances.

In computer vision, a model is widely used to describe the formation of fog image which was proposed by Koschmieder in 1924. Fig. 2 shows the model. Koschmieder presented a simple relationship between the distance $d$ of an object with intrinsic luminance $J(\boldsymbol{x})$ and its apparent luminance $I(\boldsymbol{x})$ as

$$
I(\boldsymbol{x})=t(\boldsymbol{x}) J(\boldsymbol{x})+(1-t(\boldsymbol{x})) A
$$

where $A$ denotes the intensity of the sky, and $t(\boldsymbol{x})$ describes scene transmission. Direct attenuation describes the scene radiance and its decay in the medium, while air-light from previous scattered light leads to the shift of the scene color.

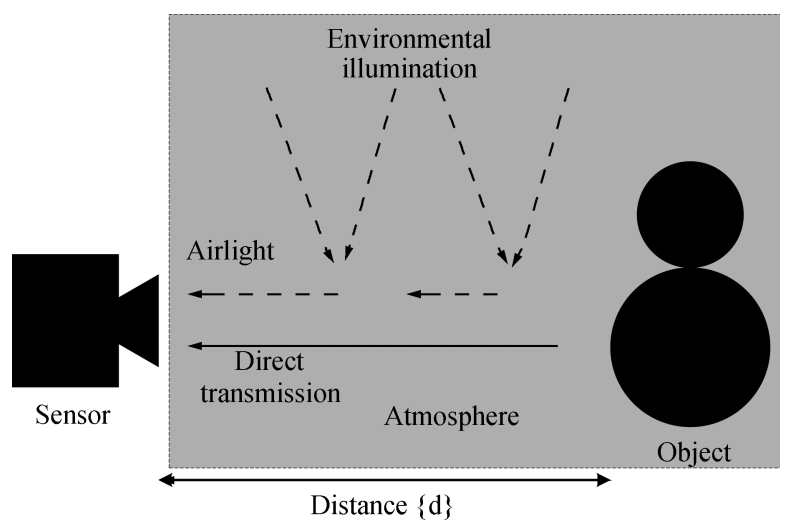

Fig. 2 Atmospheric scattering model

Particularly, in homogenous fog weather condition, scene transmission and corresponding scene distance have the following relationship:

$$
t(\boldsymbol{x})=\mathrm{e}^{-k d(\boldsymbol{x})} .
$$

From the top to the bottom in homogenous traffic fog weather background image (as the distance increases), the scene intensity will change according to the form of edge spread function, because the intensity of the road is union.

\subsection{Background generation and updating}

The main idea of the background subtraction is to collect background image information from known background and to establish the background model by utilizing background information. By comparing the input image with background model, changes of images' characteristics such as image gray levels are judged. Thereby, we can analyze the abnormal situation and segment moving objects, such as Gaussian mixture model ${ }^{[12]}$. Therefore, the establishment of background model which has been mentioned in many papers plays a crucial role in the performance of the whole detection system. However, when these methods are used, there should not be any moving object in the process of background modeling and reconstruction, and the process should take a long time. In this phase, considering time consumption, we adopt the simple algorithm RunningAvg in OpenCV to generate and update traffic scene background image. Fig. 3 shows the original scene and traffic background extraction results in three different weather conditions (sunny, snow, homogenous fog, respectively).
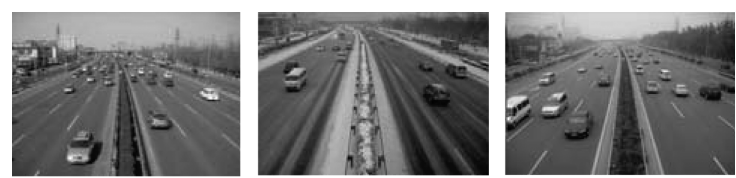

(a) Original images
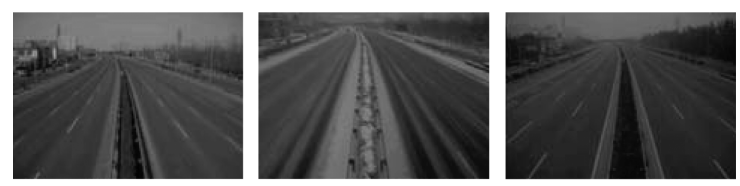

(b) Background extraction results

Fig. 3 Original images and traffic background extraction results in three different weather conditions (from left to right are sunny, snow and fog)

\subsection{Characteristics of recognition}

In order to search area for judging current weather condition, five major characteristics calculated based on gray level co-occurrence matrix (GLCM) are used. These five major characters are angular second moment (ASM), entropy (EM), contrast (COM), variance (VAR), and inverse difference moment (IDM). The detail of calculation equalities are as

$$
A S M=\sum_{i=0}^{L-1} \sum_{j=0}^{L-1} P^{2}(i, j)
$$

$$
C O N=\sum_{n=0}^{L-1} n^{2} \sum_{i=0}^{L-1} \sum_{j=0}^{L-1} P(i, j)
$$

where $|i-j|=n$.

$$
\begin{aligned}
E N T & =\sum_{i=0}^{L-1} \sum_{j=0}^{L-1} p(i, j) \log _{2} p(i, j) \\
V A R & =\sum_{i=0}^{L-1} i \sum_{j=0}^{L-1}(i-m)^{2} P(i, j)
\end{aligned}
$$

where $m$ stands for mean value of GLCM.

$$
I D M=\sum_{i=0}^{L-1} \sum_{j=0}^{L-1} \frac{P(i, j)}{1+i-j)^{2}}
$$

where $p(i, j)$ stands for pixel value in GLCM of the current frame, parameters $W$ and $H$ stand for the width and height of the current frame. These five elements constitute the characteristic vector. 


\subsection{Area search method}

In this section, we will discuss how to extract the area for recognition. Although the colors of sky and road surface are different, texture characteristics between them are similar. So we could use road model to replace sky model. Pavement, the sky and the rest of the image have different textures (Fig. 4). We assume the image size is $320 \times 240$. Three main steps for searching method are listed as follows.

Step 1. Start searching from first line of the background image: Initialize the searching rectangle size of $T_{1,1}$ by $8 \times 6$, calculate texture value in $T_{1,1}$ and save it. Slide this rectangle from left to right in the traffic background image. Every time, we only move one pixel. The rectangular regions obtained are defined as $T_{1, i}(i=1,2, \cdots, 320)$ (image size is $320 \times 240)$. All texture values in these rectangles are calculated and saved. Corresponding to each rectangle, these texture values compose characteristics vector sequences: $C_{1, i}=\left(A S M_{i}, C O N_{i}, E N T_{i}, V A R_{i}, I D M_{i}\right)$, where $i=1,2, \cdots, 320$. The characteristics vector for the prepared model in Fig. 4 is $C_{0}$. Before comparison, a constancy threshold $T$ is set. Area with minimal value is selected as candidate region:

$$
\varepsilon_{1, i}=\left|C_{1, i}-C_{0}\right|, \quad i=1,2, \cdots, 320
$$

where $\left|C_{1, i}-C_{0}\right|$ stands for absolute value of vector difference between $C_{1, i}$ and $C_{0}$. Then, the smallest one (it is assumed to be $\varepsilon_{1, j}$ ) will be compared with $T$ (threshold). The rectangle $T_{1, j}$ will be selected into our searching sequence only if $\varepsilon_{1, j}<T$, and then the algorithm turns to Step 2. Otherwise, it turns to Step 3.

Step 2. Move searching window along the longitudinal axis of the image from the top to the bottom: Like Step 1 , we assume the current line is $m$, the area we got will be marked as $T_{m, j}$. This searching window will move down from the current line until all the pixels from the top to the bottom of scene are finished.

Step 3. Reduce the height of searching window: The height of searching window will be cut to be half if $\varepsilon_{i, j}<T$. Then, turn to Step 2.

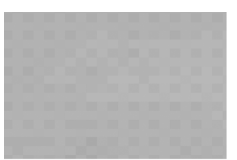

(a) Sky texture

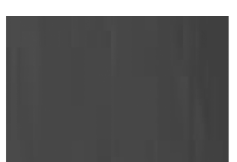

(b) Road texture

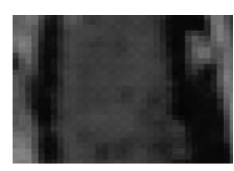

(c) Wood texture
Fig. 4 Several textures in traffic image

\subsection{Homogenous fog weather condition judgement}

Average value for each line from top to bottom could be calculated when the target area is selected. For each line,

$$
P_{i}^{A}=\frac{\sum_{k=1}^{N} P_{i, k}}{N}, \quad i=1,2, \cdots, 240
$$

where $N$ is the pixel number in the searching window. For each background image, a pixel searching sequence is got as $\left(P_{1}^{A}, P_{2}^{A}, \cdots, P_{n}^{A}\right)$. Except for some starting points in this sequence, the remaining points change according to edge spread function if it is homogenous fog.

\subsection{Recognition results}

The algorithm of weather condition recognition has been tested on three different classic weather conditions. The test traffic video sequences are captured by a fixed camera on the Beijing highway. The special weather conditions are sunny, homogenous fog, and snow. Experiment shows that the function for road about homogenous fog and homogenous fog with rain is edge spread function, while function for sunny, snowy is not. All of the extracted area is enlarged.

Fig. 5 shows the background image and their corresponding area extraction results. Fig. 6 shows the corresponding curve fitting results. It is clear that in homogenous weather condition (Fig. 5 (c)), pixel values from the top to the bottom change according to edge spread function. Other weather conditions are not in line with this fact (Fig. 5 (a) and $5(\mathrm{~b}))$.
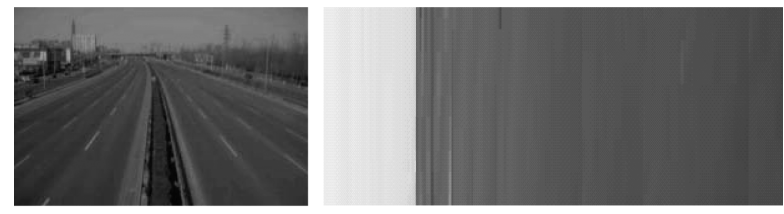

(a) Sunny background and corresponding extracted area
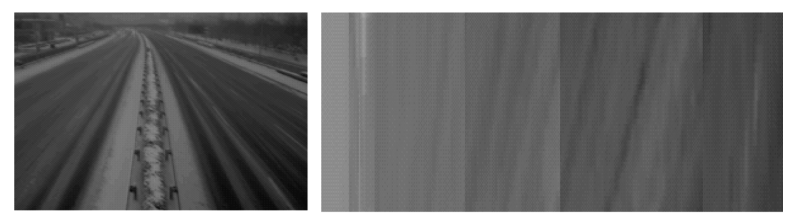

(b) Snowy background and corresponding extracted area
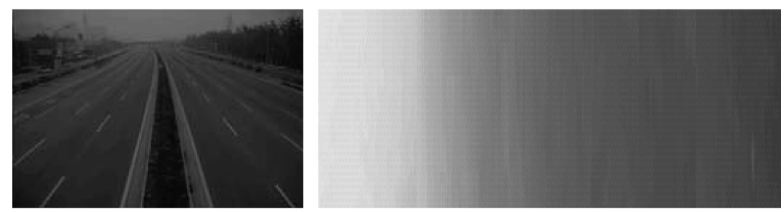

(c) Foggy background and corresponding extracted area

Fig. 5 Background image and corresponding extraction area which are enlarged

Fig. 7 shows another three different background images and their corresponding area extraction results. These three scenes are called SA, SB and SC. SA and SB stand for common fog scene. SC stands for fog mixed with rain. It is clear that the three images are in line with the form of edge spread function (Fig. 8). We take rain as noise. From this, we can see that our algorithm can deal with the noise interference.

\section{Calibration model}

Image depth and vehicle distance cannot be obtained by using just a single camera in ordinary scene. But it could be overcome by the hypothesis that the road is a flat plane. In this part, we deduced calibration formula from monocular liner model and dark channel prior. 


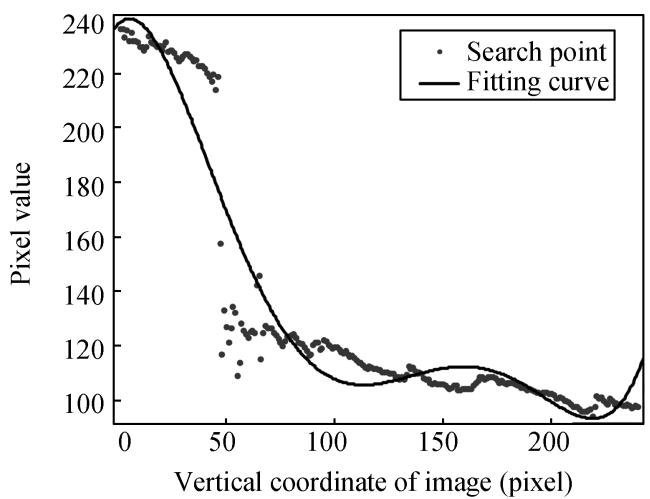

(a) Fitting curve of the extracted area in sunny condition

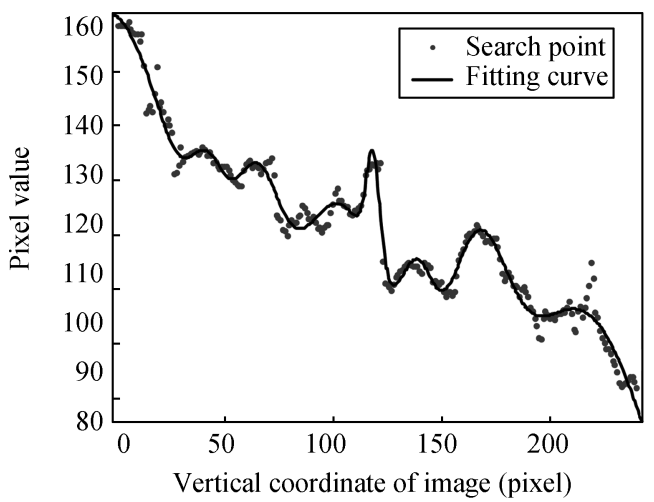

(b) Fitting curve of the extracted area in snow condition

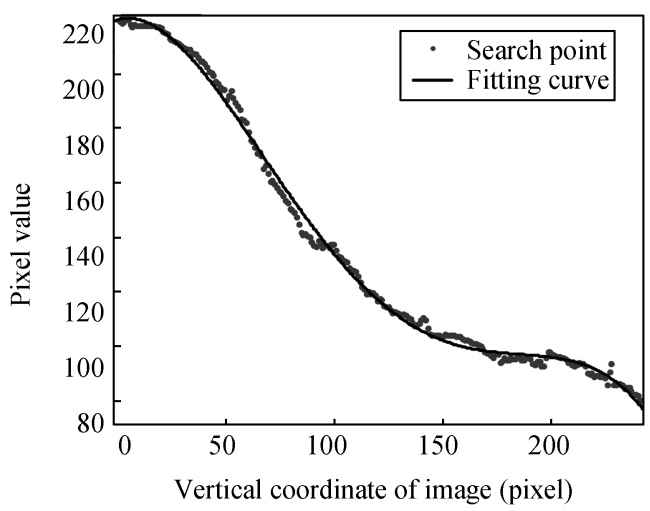

(c) Fitting curve of the extracted area in fog condition

Fig. 6 Function fitting results corresponding to Fig. 5

\subsection{Three ordinary coordinate systems}

Image coordinate system, camera coordinate system and world coordinate system are defined as follows.

Image coordinates expressed by pixel unit: In Cartesian coordinate system, $u, v$ stand for the image plane, the coordinate $(u, v)$ of each pixel describes the number of rows and columns where the pixel point is located at.

Image coordinates expressed by physical unit: Because $(u, v)$ does not express the position on the image by physical unit, it needs to build the image coordinates expressed by physical unit (such as millimeter). In Fig. 9, $O_{1}$ is the origin of the image coordinate, $x$ and $y$ axes are parallel with $u$ and $v$, respectively. If the coordinate of $O_{1}$ is $\left(u_{0}, v_{0}\right)$ in the image coordinate, the physical size of every pixel point is $d x$ and $d y$ in the orientation of $x$ and $y$ axis. $O_{c} O_{1}$ is the focus of camera (marked as $f$ ).

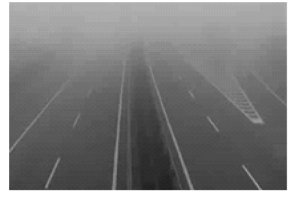

(a) SA background and extracted area

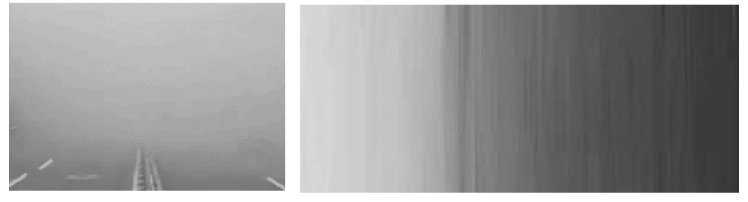

(b) SB background and extracted area

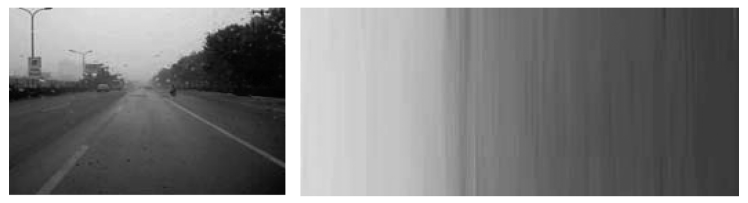

(c) SC background and extracted area

Fig. 7 Three scenes and corresponding extraction area which are enlarged

Camera coordinates system: Cartesian coordinate system is composed of $O_{c}, X_{c}, Y_{c}$ and $Z_{c} . X_{c}$ and $Y_{c}$ axes are parallel with $x$ and $y$ axes, respectively.

World coordinates system: Any position in scene could be selected to describe the position of the camera. We call it world coordinates system which is composed of $X_{w}, Y_{w}$ and $Z_{w}$.

Relationship between world coordinates system and image coordinates system: By applying the pinhole model for the camera, a point with three-dimensional coordinates $\left(X_{C}, Y_{C}, Z_{C}\right)$ within the camera reference system is projected onto the image plane in accordance with the following expression:

$$
Z_{c}\left[\begin{array}{l}
u \\
v \\
1
\end{array}\right]=
$$

$$
\begin{aligned}
& {\left[\begin{array}{ccc}
\frac{1}{d x} & 0 & u_{0} \\
0 & \frac{1}{d y} & v_{0} \\
0 & 0 & 1
\end{array}\right]\left[\begin{array}{llll}
f & 0 & 0 & 0 \\
0 & f & 0 & 0 \\
0 & 0 & 1 & 0
\end{array}\right] \times} \\
& {\left[\begin{array}{cc}
R & t \\
0^{T} & 1
\end{array}\right]\left[\begin{array}{c}
X_{w} \\
Y_{w} \\
Z_{w} \\
1
\end{array}\right]=} \\
& {\left[\begin{array}{cccc}
a_{x} & 0 & u_{0} & 0 \\
0 & a_{y} & v_{0} & 0 \\
0 & 0 & 1 & 0
\end{array}\right] \times}
\end{aligned}
$$




$$
\begin{aligned}
& {\left[\begin{array}{cc}
R & t \\
0^{\mathrm{T}} & 1
\end{array}\right] \times\left[\begin{array}{c}
X_{w} \\
Y_{w} \\
Z_{w} \\
1
\end{array}\right]=} \\
& M_{1} M_{2} X_{w}= \\
& M X_{w}
\end{aligned}
$$

where $a_{x}=\frac{f}{d x}, a_{y}=\frac{f}{d y}$, and $M$ is $3 \times 4$ matrix.

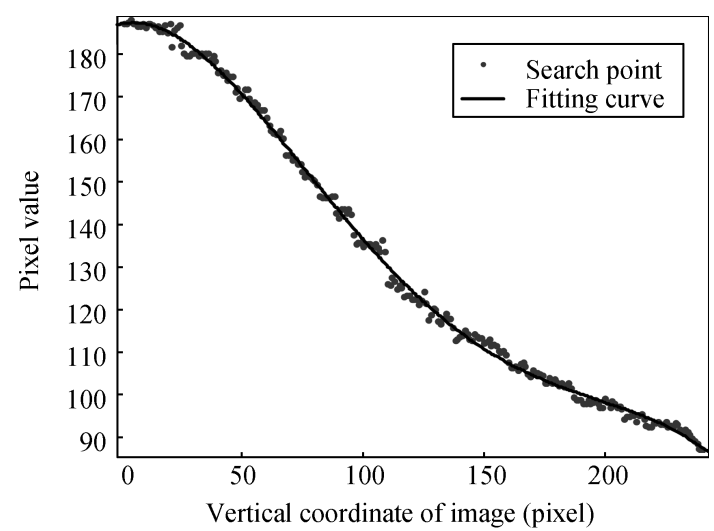

(a) Fitting result for extracting area in SA

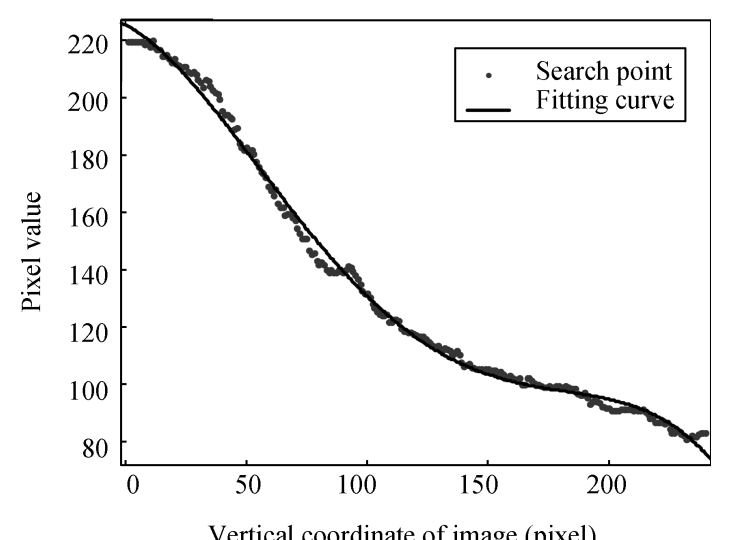

(b) Fitting result for extracting area in $\mathrm{SB}$

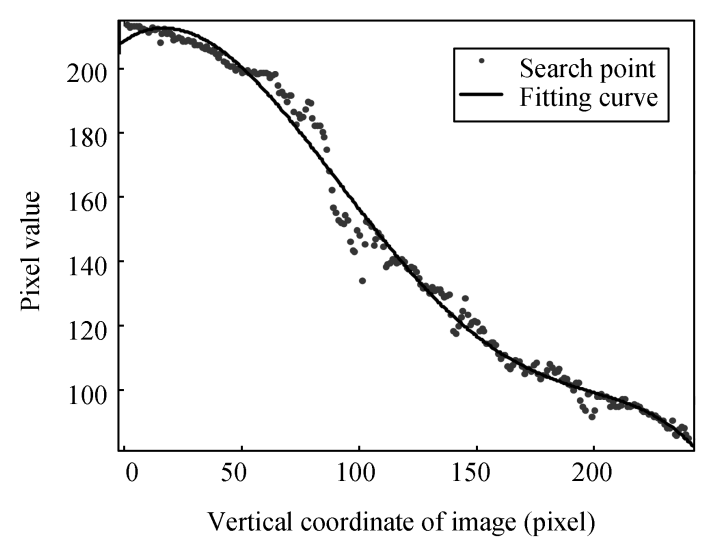

(c) Fitting result for extracting area in $\mathrm{SC}$

Fig. 8 Three scene functions fitting results corresponding to Fig. 7

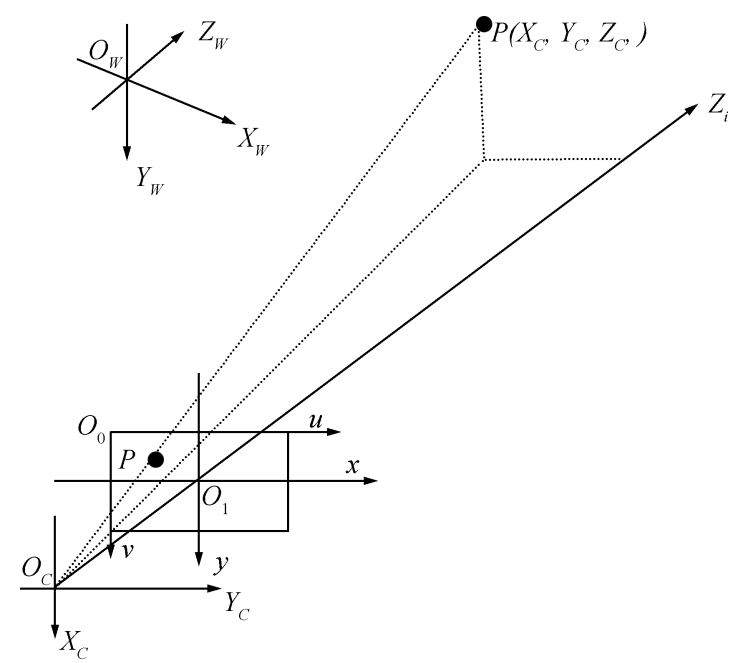

Fig. 9 The world coordinate, camera coordinate and image coordinate

\subsection{Particular monocular model for veloc- ity detection}

To provide a context for velocity estimation algorithm, we indicate a monocular model. In our model, no painted lines exist. Lines $L_{1}$ and $L_{2}$ stand for the edges of the road. They are only used to delineate traffic lane but are not detected in our algorithm. The geometry of our camera and roadway scene is shown in Fig. 10. We model the scene as a set of parallel lines viewed through a simple pinhole camera, assuming that the camera is located at a height $h$ (which we have known ahead) above the ground plane. The camera is oriented at a pan angle $\theta$ with respect to the road, and tilt (down) angle $\varphi$ such that a point in the earth coordinate system $\left(X_{W}, Y_{W}, Z_{W}\right)$ is transformed into the camera coordinate system $\left(X_{C}, Y_{C}, Z_{C}\right)$ by only a translation and a rotation. The camera is oriented along the negative $Z_{C}$ axis (hence the negative sign associated with the focal length), and its line of sight intersects the ground plane at a distance $F=h \times \csc \varphi$ away.

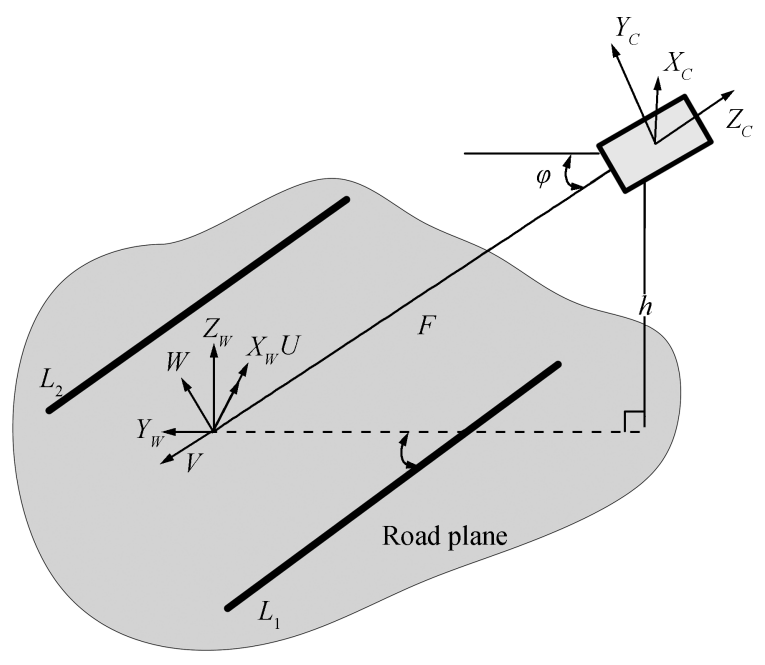

Fig. 10 Traffic camera model

This model is accurate if the road is assumed to be planar 
and the rotation of the ground plane about the $Z_{C}$ axes is negligible. There is theoretically less than $10 \%$ bias in distance measurements for road grades of $2 \%$ based on the analysis with a full (three-angle) camera model. The theoretical bias will be smaller when using the reduced (twoangle) camera model. The following equalities describe the perspective projection of points in the pinhole camera's coordinate system onto the image coordinate plane, given a focal length $f$ :

$$
\begin{aligned}
& \frac{u-u_{0}}{\alpha_{x}}=-\frac{X_{C}}{Z_{C}} \\
& \frac{v-v_{0}}{\alpha_{y}}=-\frac{Y_{C}}{Z_{C}}
\end{aligned}
$$

where $a_{x}=\frac{f}{d x}$, and $a_{y}=\frac{f}{d y}$.

Starting with the ground-plane $X_{W}-Y_{W}-Z_{W}$ coordinate system, we develop expressions for the $X_{C}-Y_{C}-Z_{C}$ coordinates. First, the $U-V-W$ system is obtained by rotating an angle $\varphi$ around the $X_{W}$ axis.

$$
\begin{aligned}
& U=X_{W} \\
& V=Y_{W} \times \cos \varphi-Z_{W} \times \sin \varphi \\
& W=Y_{W} \times \sin \varphi+Z_{W} \times \cos \varphi
\end{aligned}
$$

These expressions are further simplified because objects are assumed to lie on the ground plane denoting $Z_{C}$ coordinate. Next, there is a displacement to obtain the cameracentered coordinates $X_{C}-Y_{C}-Z_{C}$.

$$
\begin{aligned}
& X_{C}=U=X_{W} \\
& Y_{C}=W=Y_{W} \times \sin \varphi \\
& Z_{C}=-V-F=-Y_{W} \times \cos \varphi-F .
\end{aligned}
$$

Applying (1) yields

$$
\begin{array}{r}
u-u_{0}=-\alpha_{x} \frac{X_{C}}{Z_{C}}=-\alpha_{x} \frac{X_{W}}{-Y_{W} \times \cos \varphi-F} \\
v-v_{0}=-\alpha_{y} \frac{Y_{C}}{Z_{C}}=-\alpha_{y} \frac{Y_{W} \times \sin \varphi}{-Y_{W} \times \cos \varphi-F} \\
u-u_{0}=-\alpha_{x} \frac{X_{C}}{Z_{C}}=\frac{\alpha_{x} \times X_{W}}{F+Y_{W} \times \cos \varphi} \\
v-v_{0}=-\alpha_{y} \frac{Y_{C}}{Z_{C}}=\frac{\alpha_{y} \times Y_{W} \times \sin \varphi}{F+Y_{W} \times \cos \varphi} .
\end{array}
$$

From (14) and (16), we could get

$$
\begin{gathered}
\frac{\sin \varphi \times \alpha_{y}}{v-v_{0}}=\frac{F+v \times \cos \varphi}{Y_{W}} \\
Y_{W}=\frac{h \times\left(v-v_{0}\right)}{\sin \varphi \times\left[\sin \varphi \times \alpha_{y}-\cos \varphi \times\left(v-v_{0}\right)\right]} .
\end{gathered}
$$

Substitute (19) into (14), $Z_{C}$ could be got as

$$
Z_{C}=\frac{-h \times\left(v-v_{0}\right) \times \cos \varphi}{\sin \varphi \times\left[\sin \varphi \times \alpha_{y}-\cos \varphi \times\left(v-v_{0}\right)\right]}-\frac{h}{\sin \varphi} .
$$

Equality (20) combined with dark channel prior theory will be used to calculate camera parameters. Details will be discussed lately.

\subsection{Traffic road surface detection based on activity map}

Generating the activity map: An activity map is used to identify both the location and intensity of vehicular motion $^{[10]}$. Inactive lanes are thus excluded from the velocity estimation. Effective area could only be used for camera calibration. We assume that pixel values change over time as the vehicles pass through the image and that the only significant motion in the image is due to the vehicles only. We generate the activity map by calculating the expected value of the absolute intensity difference $D$ between two frames $I_{i}$ and $I_{i+1}$. Each image frame is smoothed with a $3 \times 3$ boxcar kernel to remove JPEG artifacts.

Such a summation of an image sequence resembles a concept presented in [13] where the authors described how to generate an activity map with binary images obtained by the threshold. They used the map to obtain very rough lane masks using morphological operators. Our method does not require us to choose a threshold for image binarized and is more appropriate for the task of extracting the traffic lanes.

Activity map results: Fig. 12 shows activity map result for homogenous fog weather condition corresponding to Fig. 11. Points within the gray area must be on the traffic plane. These points will have the opportunity to be selected to calculate camera parameters. Area with white color stands for the traffic road surface. So it is easy to distinguish points on the road or points out of the road surface.

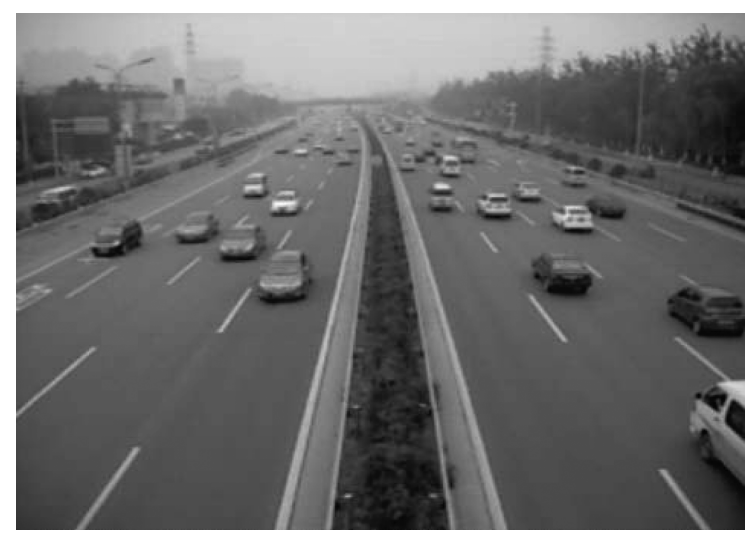

Fig. 11 Original image

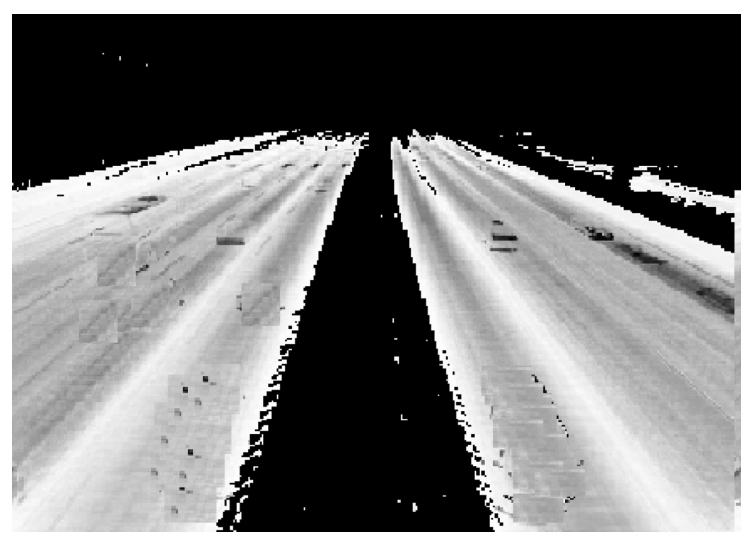

Fig. 12 Activity map extraction results 


\subsection{Dark channel prior theory}

Three steps are needed for traditional binocular vision algorithm, the stage of camera calibration, stage of matching, and the stage of depth calculation. This traditional model neglects the scene distance implicit in light travel model in the air. Firstly we introduce dark channel prior theory.

Definition of dark prior: In the foggy image, the strength of the dark pixels will be increased by atmospheric light. These dark pixels can be used to evaluate the value of transmission. Formally, for an image $J(\boldsymbol{x})$, we define

$$
J^{\operatorname{dark}}(\boldsymbol{x})=\min _{c \in\{R, G, B\}}\left(\min _{\boldsymbol{y} \in \Omega(\boldsymbol{x})}\left(J^{c}(\boldsymbol{y})\right)\right)
$$

where $J^{c}(\boldsymbol{y})$ is a color channel of $J$, and $\Omega(\boldsymbol{x})$ is a local patch centered at vector $\boldsymbol{x}$. Observation says that except for the sky region, the intensity of $J^{\text {dark }}(\boldsymbol{x})$ is low and tends to be zero, if $J(\boldsymbol{x})$ is a fog-free outdoor image. We call $J^{\text {dark }}(\boldsymbol{x})$ the dark channel of $J$, and we call the above statistical observation or knowledge the dark channel prior.

Estimating scene transmission: Taking the min operation in the local patch on light traveling equality (1), we have

$$
\min _{\boldsymbol{y} \in \Omega(\boldsymbol{x})} I^{c}(\boldsymbol{y})=t(\boldsymbol{x}) \min _{\boldsymbol{y} \in \Omega(\boldsymbol{x})} J^{c}(\boldsymbol{y})+(1-t(\boldsymbol{x})) A^{c} .
$$

It can be noticed that the min operation is performed on three color channels independently. This equality is equivalent to

$$
\min _{\boldsymbol{y} \in \Omega(\boldsymbol{x})} \frac{I^{c}(\boldsymbol{y})}{A^{c}}=t(\boldsymbol{x}) \min _{\boldsymbol{y} \in \Omega(\boldsymbol{x})} \frac{J^{c}(\boldsymbol{y})}{A^{c}}+(1-t(\boldsymbol{x})) .
$$

Then, take the min operation among three color channels on the above equation, we obtain

$$
\min _{c} \min _{\boldsymbol{y} \in \Omega(\boldsymbol{x})} \frac{I^{c}(\boldsymbol{y})}{A^{c}}=t(\boldsymbol{x}) \min _{c} \min _{\boldsymbol{y} \in \Omega(\boldsymbol{x})} \frac{J^{c}(\boldsymbol{y})}{A^{c}}+(1-t(\boldsymbol{x})) .
$$

According to the dark channel prior, the dark channel $J^{\text {dark }}(\boldsymbol{x})$ of the fog-free radiance $J$ should tend to be zero as

$$
J^{\operatorname{dark}}(\boldsymbol{x})=\min _{c} \min _{\boldsymbol{y} \in \Omega(\boldsymbol{x})} J^{c}(\boldsymbol{y})=0 .
$$

As $A^{c}$ is always positive, this leads to

$$
J^{\text {dark }}(\boldsymbol{x})=\min _{c} \min _{\boldsymbol{y} \in \Omega(\boldsymbol{x})} \frac{J^{c}(\boldsymbol{y})}{A^{c}}=0 .
$$

Putting (26) into (24), we can estimate the transmission $t(\boldsymbol{x})$ simply by

$$
t(\boldsymbol{x})=1-\min _{c} \min _{\boldsymbol{y} \in \Omega(\boldsymbol{x})} \frac{I^{c}(\boldsymbol{y})}{A^{c}} .
$$

The dark channel prior is not a good prior for the sky regions. Fortunately, the color of the sky is usually very similar to the atmospheric light $A$ in a hazy image and we have

$$
\min _{c}\left(\min _{\boldsymbol{y} \in \Omega(\boldsymbol{x})}\left(\frac{I^{c}(\boldsymbol{y})}{A^{c}}\right)\right) \rightarrow 1 .
$$

Put (28) into (27), we can get $t(\boldsymbol{x}) \rightarrow 0$.

Since the sky is at infinity and tends to have zero transmission, (27) gracefully handles both sky regions and nonsky regions. We do not need to separate the sky regions beforehand.
Results of transmission about the sense: Fig. 13 shows the transmission image in three different weather conditions. It takes about $4 \mathrm{~ms}$ for one image processing on a $\mathrm{PC}$ with a $1.0 \mathrm{GHz}$ Intel Pentium 4 processor. Transmission image distribution emphasizes more about lane and omits some details as compared to the original image.
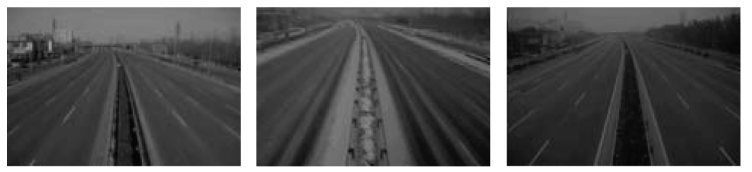

(a) Background images in sun, snow and fog
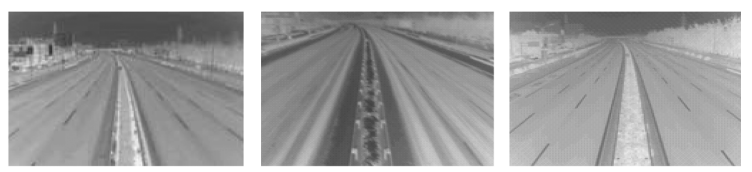

(b) Transition images in sun, snow and fog

Fig. 13 Transition images corresponding to three different weather conditions (from left to right are sun, snow and fog)

\subsection{Calibration formula based on dark channel prior and camera linear model}

Applying (2), we could get

$$
d(\boldsymbol{x})=\frac{\ln t(\boldsymbol{x})}{-k} .
$$

Distance between any point in traffic surface road and the camera could be calculated by its camera coordinate in camera coordinate system, i.e.,

$$
\begin{aligned}
& d(\boldsymbol{x})=\sqrt{X_{C}^{2}+Y_{C}^{2}+Z_{C}^{2}} \\
& d^{2}(\boldsymbol{x})=X_{C}^{2}+Y_{C}^{2}+Z_{C}^{2} .
\end{aligned}
$$

Divide both sides of (31) by $Z_{C}^{2}$ :

$$
\frac{X_{C}^{2}}{Z_{C}^{2}}+\frac{Y_{C}^{2}}{Z_{C}^{2}}+1=\frac{d^{2}(\boldsymbol{x})}{Z_{C}^{2}} .
$$

Substitute (11), (12) into (32), we could get

$$
\left(\frac{u-u_{0}}{\alpha_{x}}\right)^{2}+\left(\frac{v-v_{0}}{\alpha_{y}}\right)^{2}+1=\frac{d^{2}(\boldsymbol{x})}{Z_{C}^{2}} .
$$

In order to eliminate the unknown parameter $k$, we introduce two points on the road surface at the same time:

$$
\begin{aligned}
& \left(\frac{u_{1}-u_{0}}{\alpha_{x}}\right)^{2}+\left(\frac{v_{1}-v_{0}}{\alpha_{y}}\right)^{2}+1=\frac{d^{2}\left(\boldsymbol{x}_{1}\right)}{Z_{1 C}^{2}} \\
& \left(\frac{u_{2}-u_{0}}{\alpha_{x}}\right)^{2}+\left(\frac{v_{2}-v_{0}}{\alpha_{y}}\right)^{2}+1=\frac{d^{2}\left(\boldsymbol{x}_{2}\right)}{Z_{2 C}^{2}}
\end{aligned}
$$

where $d\left(\boldsymbol{x}_{1}\right)=\frac{\ln t\left(\boldsymbol{x}_{1}\right)}{-k}, d\left(\boldsymbol{x}_{2}\right)=\frac{\ln t\left(\boldsymbol{x}_{2}\right)}{-k}, \boldsymbol{x}_{1}=\left(u_{1}, v_{1}\right)$, and $\boldsymbol{x}_{2}=\left(u_{2}, v_{2}\right)$ stand for two different points on the road surface. Divide both sides of (34) by (35), respectively:

$$
\frac{\left(\frac{u_{1}-u_{0}}{\alpha_{x}}\right)^{2}+\left(\frac{v_{1}-v_{0}}{\alpha_{y}}\right)^{2}+1}{\left(\frac{u_{2}-u_{0}}{\alpha_{x}}\right)^{2}+\left(\frac{v_{2}-v_{0}}{\alpha_{y}}\right)^{2}+1}=\left[\frac{\ln t\left(\boldsymbol{x}_{1}\right)}{\ln t\left(\boldsymbol{x}_{2}\right)}\right]^{2} \times \frac{Z_{2 C}^{2}}{Z_{1 C}^{2}} \text {. }
$$


Take (20) into (28), we obtain

$$
\begin{aligned}
& \frac{\left(\frac{u_{1}-u_{0}}{\alpha_{x}}\right)^{2}+\left(\frac{v_{1}-v_{0}}{\alpha_{y}}\right)^{2}+1}{\left(\frac{u_{2}-u_{0}}{\alpha_{x}}\right)^{2}+\left(\frac{v_{2}-v_{0}}{\alpha_{y}}\right)^{2}+1}= \\
& {\left[\frac{\ln t\left(\boldsymbol{x}_{1}\right)}{\ln t\left(\boldsymbol{x}_{2}\right)}\right]^{2} \times\left[\frac{\sin \varphi \times \alpha_{y}-\cos \varphi \times\left(v_{1}-v_{0}\right)}{\sin \varphi \times \alpha_{y}-\cos \varphi \times\left(v_{2}-v_{0}\right)}\right]^{2} .}
\end{aligned}
$$

For most cameras, $\alpha_{x}=\alpha_{y}=\alpha$, (37) turns to

$$
\begin{aligned}
& \frac{\left(\frac{u_{1}-u_{0}}{\alpha}\right)^{2}+\left(\frac{v_{1}-v_{0}}{\alpha}\right)^{2}+1}{\left(\frac{u_{2}-u_{0}}{\alpha}\right)^{2}+\left(\frac{v_{2}-v_{0}}{\alpha}\right)^{2}+1}= \\
& {\left[\frac{\ln t\left(\boldsymbol{x}_{1}\right)}{\ln t\left(\boldsymbol{x}_{2}\right)}\right]^{2} \times\left[\frac{\sin \varphi \times \alpha-\cos \varphi \times\left(v_{1}-v_{0}\right)}{\sin \varphi \times \alpha-\cos \varphi \times\left(v_{2}-v_{0}\right)}\right]^{2}}
\end{aligned}
$$

i.e.,

$$
\begin{aligned}
& \frac{\left(u_{1}-u_{0}\right)^{2}+\left(v_{1}-v_{0}\right)^{2}+\alpha^{2}}{\left(u_{2}-v_{0}\right)^{2}+\left(v_{2}-v_{0}\right)^{2}+\alpha^{2}}= \\
& {\left[\frac{\ln t\left(\boldsymbol{x}_{1}\right)}{\ln t\left(\boldsymbol{x}_{2}\right)}\right]^{2} \times\left[\frac{\sin \varphi \times \alpha-\cos \varphi \times\left(v_{1}-v_{0}\right)}{\sin \varphi \times \alpha-\cos \varphi \times\left(v_{2}-v_{0}\right)}\right]^{2} .}
\end{aligned}
$$

Equality (39) is the base formula used in this article, camera parameters $\left(\alpha, u_{0}, v_{0}, \varphi\right)$ are all unknown. This equality which is very complex is a trigonometric equation mixed with four unknown quadratic functions. It is difficult to be solved unless we could select several points in the road surface to simplify this equation. We notice that if we select two pairs of points in the same line, (39) will turn to be linear function, the detailed deduction process is as

$$
\begin{aligned}
& \left(u_{1}-u_{0}\right)^{2}+\left(v_{1}-v_{0}\right)^{2}+\alpha^{2}= \\
& {\left[\frac{\ln t\left(\boldsymbol{x}_{1}\right)}{\ln t\left(\boldsymbol{x}_{2}\right)}\right]^{2}\left[\left(u_{2}-u_{0}\right)^{2}+\left(v_{2}-v_{0}\right)^{2}+\alpha^{2}\right]} \\
& \left(u_{3}-u_{0}\right)^{2}+\left(v_{3}-v_{0}\right)^{2}+\alpha^{2}= \\
& {\left[\frac{\ln t\left(\boldsymbol{x}_{3}\right)}{\ln t\left(\boldsymbol{x}_{4}\right)}\right]^{2}\left[\left(u_{4}-u_{0}\right)^{2}+\left(v_{4}-v_{0}\right)^{2}+\alpha^{2}\right]}
\end{aligned}
$$

where $\boldsymbol{x}_{3}=\left(u_{3}, v_{3}\right)$ and $\boldsymbol{x}_{4}=\left(u_{4}, v_{4}\right)$. Points $\boldsymbol{x}_{3}$ and $\boldsymbol{x}_{4}$ are in the same line of the road surface. Let $\left[\frac{\ln t\left(\boldsymbol{x}_{1}\right)}{\ln t\left(\boldsymbol{x}_{2}\right)}\right]^{2}=A$ and $\left[\frac{\ln t\left(\boldsymbol{x}_{3}\right)}{\ln t\left(\boldsymbol{x}_{4}\right)}\right]^{2}=B$, take them into (40) and (41), we can get

$$
u_{0}^{2}-\frac{2\left(u_{1}-A u_{2}\right)}{1-A} u_{0}+v_{0}^{2}-2 v_{1} v_{0}+\frac{u_{1}^{2}-A u_{2}^{2}}{1-A}+\alpha^{2}+v_{1}^{2}=0
$$$$
u_{0}^{2}-\frac{2\left(u_{3}-B u_{4}\right)}{1-B} u_{0}+v_{0}^{2}-2 v_{3} v_{0}+\frac{u_{3}^{2}-B u_{4}^{2}}{1-B}+\alpha^{2}+v_{3}^{2}=0 \text {. }
$$

Subtract (42) from (43), we can get

$$
\begin{aligned}
& 2\left[\frac{\left(u_{3}-B u_{4}\right)}{1-B}-\frac{\left(u_{1}-B u_{2}\right)}{1-A}\right] u_{0}+2\left(v_{3}-v_{1}\right) v_{0}+ \\
& \frac{u_{1}^{2}-A u_{2}^{2}}{1-A}-\frac{u_{3}^{2}-B u_{4}^{2}}{1-B}+v_{1}^{2}-v_{3}^{2}=0 .
\end{aligned}
$$

Equality (44) is linear. We find that four points (two pairs) which are located at the same line could generate one linear equality. In order to solve the liner equality, we need another liner equality. Similarly, we select another four points (two pairs) on road surface, they are $\boldsymbol{x}_{5}=\left(u_{5}, v_{5}\right)$, $\boldsymbol{x}_{6}=\left(u_{6}, v_{6}\right), \boldsymbol{x}_{7}=\left(u_{7}, v_{7}\right)$, and $\boldsymbol{x}_{8}=\left(u_{8}, v_{8}\right)$, where $\boldsymbol{x}_{5}=$ $\left(u_{5}, v_{5}\right), \boldsymbol{x}_{6}=\left(u_{6}, v_{6}\right)$ are in the same line, $\boldsymbol{x}_{7}=\left(u_{7}, v_{7}\right)$, $\boldsymbol{x}_{8}=\left(u_{8}, v_{8}\right)$ are in the same line. So these four points generate another liner equality as

$$
\begin{aligned}
& 2\left[\frac{\left(u_{7}-D u_{8}\right)}{1-D}-\frac{\left(u_{5}-D u_{6}\right)}{1-C}\right] u_{0}+2\left(v_{7}-v_{5}\right) v_{0}+ \\
& \frac{u_{5}^{2}-C u_{6}^{2}}{1-C}-\frac{u_{7}^{2}-D u_{8}^{2}}{1-D}+v_{5}^{2}-v_{7}^{2}=0
\end{aligned}
$$

where $C=\left[\frac{\ln t\left(\boldsymbol{x}_{5}\right)}{\ln t\left(\boldsymbol{x}_{\mathbf{6}}\right)}\right]$ and $D=\left[\frac{\ln t\left(\boldsymbol{x}_{7}\right)}{\ln t\left(\boldsymbol{x}_{8}\right)}\right]$. Camera parameters $u_{0}$ and $v_{0}$ are calculated through equation (44) and (45). Parameter $\alpha$ is got by taking the calculation results of $u_{0}$ and $v_{0}$ into (40). Finally, take $u_{0}, v_{0}, \alpha$ into (39), and parameter $\varphi$ is got.

\subsection{Point selection method for equation generating}

To get camera parameters, the following three steps will be undertaken as given by Fig. 14. These three steps can also detect and delete noise on road surface.

Step 1. Calculation of $u_{0}$ and $v_{0}$ : We extract two pairs of points in the road plane to calculate parameters $u_{0}$ and $v_{0}$. From the top to the bottom of the traffic road surface, for each row, we select two points that have maximum transmission difference value. They are marked as $\left(P_{i, 1}, P_{i, 2}\right)(i=m, m+1, \cdots, 240)$, where $m$ is the vertical coordinates starting line of the traffic road surface and the image size is $320 \times 240$. In all these point pairs, select two pairs that have the largest transmission difference. We may mark them as $\left(P_{s, 1}, P_{s, 2}\right)\left(P_{t, 1}, P_{t, 2}\right)$, where $s$ and $t$ stand for line number. We mark these four points by $A, B, C, D$ in Fig. 14. By using these two point pairs (points), we could get $u_{0}$ and $v_{0}$ based on (44).

Step 2. Calculation for $\alpha$ and $\varphi$ : Based on the calculation results in Step 1, in lines $s$ and $t$, we select two point pairs, separately. We call these four points as $E, F$, $G, H$, in Fig. 14. By using (39), we could get another two parameters $\alpha$ and $\varphi$.

Step 3. Multi frames for average parameters: Through Steps 1 and 2, all the parameters could be got. One correction is over, however it is necessary to calculate average values through multi frames.

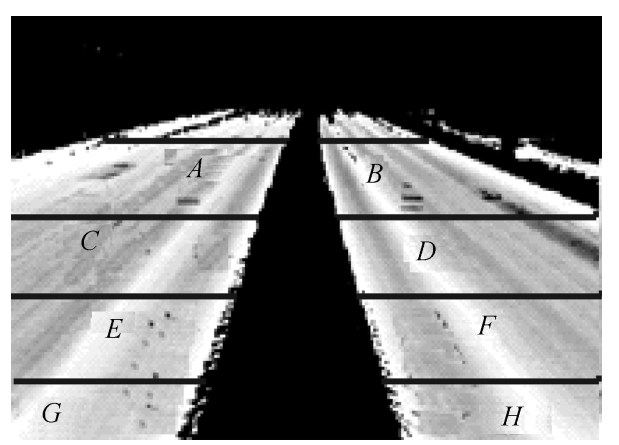

Fig. 14 Point selection method for generating equation 


\section{Distance and velocity calculation}

Once calibrated, the camera coordinates and world coordinate could be got as

$$
\begin{aligned}
X_{C}= & \frac{h \times\left(u-u_{0}\right)}{\left[\sin \phi \times \alpha-\cos \phi \times\left(v-v_{0}\right)\right]} \\
Y_{C}= & \frac{h \times\left(v-v_{0}\right)}{\sin \phi \times \alpha-\cos \phi \times\left(v-v_{0}\right.} \\
Z_{C}= & \frac{-h \times\left(v-v_{0}\right) \times \cos \phi}{\sin \phi} \times \\
& {\left[\sin \phi \times \alpha-\cos \phi \times\left(v-v_{0}\right)\right]-\frac{h}{\sin \phi} } \\
X_{W}= & \frac{h \times\left(u-u_{0}\right)}{\sin \phi \times \alpha-\cos \phi \times\left(v-v_{0}\right)} \\
Y_{W}= & \frac{h \times\left(v-v_{0}\right)}{\sin \phi \times\left[\sin \phi \times \alpha-\cos \phi \times\left(v-v_{0}\right)\right]} \\
Z_{W}= & 0 .
\end{aligned}
$$

Distance between points in the road surface and the origin of world coordinate system is

$$
D_{v}=\sqrt{X_{C}^{2}+Y_{C}^{2}+Z_{C}^{2}} .
$$

The traveling distance for moving vehicles on the road surface is got by its distance difference, and the corresponding velocity is got by dividing its consuming time as

$$
V_{i}=\frac{\Delta D_{v}^{i}}{T_{i}}
$$

where $\Delta D_{v}^{i}$ stands for traveling displacement difference, and $T_{i}$ stands for the corresponding consuming time.

\section{Results and discussion}

In our experiment, two parts are included. In the first part, we only use fog weather video sequences to calibrate the camera. Point extraction results for three frames and ultimate average calibration results are listed. Fig. 5 shows the result compared with other algorithms. Detailed parameter calibration results compared with other three traffic calibration methods from frame 500 to frame 1000 are given in Fig. 16. We compare our algorithm with the method based on vanishing point ${ }^{[14]}$, method based on corner detection ${ }^{[15]}$, and the classic 6 -point camera calibration method for liner camera model. In the second part, we give 9 vehicles velocity calculation results corresponding to three different weather conditions. This camera calibration and velocity estimation algorithm is tested by one affixed video frames captured from the same scene by the same angle in Beijing. This video frame also includes two parts. The first part which has 1000 frames is captured in homogenous fog weather condition and is only used for camera calibration. Another part is our testing sequences, each of which has 1500 frames (500 frames for each weather condition). Because the background generation and updating algorithm consumes much time to refresh the background, we execute parameter calculation algorithm after 500 frames in the video frames of the first part. Tables $1-3$ show the point extraction results for the generating parameter calculation equality and the parameter calculation results corresponding to Fig. 15 (homogenous fog weather condition). For the sake of convenience, all points selected for equation generation are named as $A, B, C, D, E, F, G$ and $H$. Results for three frames (frame 500, frame 800 and frame 1000) and their average parameter values are listed in Table 4 .

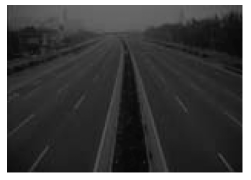

(a) Frame 500

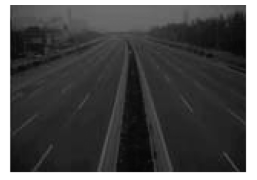

(b) Frame 800

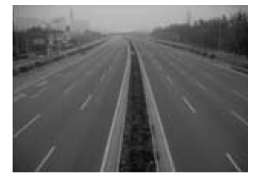

(c) Frame 1000
Fig. 15 Three background images in homogenous fog weather condition

Table 1 Point extraction results for frame 600 (Fog)

\begin{tabular}{cccccc}
\hline Point & $u$ & $v$ & Point & $u$ & $v$ \\
\hline$A$ & 34 & 84 & $E$ & 95 & 84 \\
$B$ & 250 & 84 & $F$ & 224 & 84 \\
$C$ & 37 & 195 & $G$ & 12 & 195 \\
$D$ & 266 & 195 & $H$ & 232 & 195 \\
\hline
\end{tabular}

Table 2 Point extraction results for frame 800 (Fog)

\begin{tabular}{cccccc}
\hline Point & $u$ & $v$ & Point & $u$ & $v$ \\
\hline$A$ & 69 & 80 & $E$ & 52 & 80 \\
$B$ & 230 & 80 & $F$ & 120 & 80 \\
$C$ & 52 & 116 & $G$ & 238 & 116 \\
$D$ & 83 & 116 & $H$ & 264 & 195 \\
\hline
\end{tabular}

Table 3 Point extraction results for frame 1000 (Fog)

\begin{tabular}{cccccc}
\hline Point & $u$ & $v$ & Point & $u$ & $v$ \\
\hline$A$ & 8 & 160 & $E$ & 10 & 160 \\
$B$ & 34 & 160 & $F$ & 29 & 160 \\
$C$ & 280 & 180 & $G$ & 12 & 180 \\
$D$ & 316 & 180 & $H$ & 56 & 180 \\
\hline
\end{tabular}

Table 4 Camera parameter calibration result (Fog)

\begin{tabular}{ccccc}
\hline Frame & $u_{0}$ & $v_{0}$ & $\varphi$ & $\alpha$ \\
\hline 600 & 149 & 118 & $11.8^{\circ}$ & 369.2 \\
800 & 150 & 118 & $11.6^{\circ}$ & 370.3 \\
1000 & 149 & 120 & $12.1^{\circ}$ & 370.4 \\
Average & 149.4 & 119.2 & $12.1^{\circ}$ & 369.6 \\
\hline
\end{tabular}

Detailed parameter comparison results are shown in Table 5 and Fig. 16. We take results got by 6 -point as the real data. From the experiments, we could see that the coefficient in our algorithm is much ideal and parameters are closer to standard values. The calibration method based on motion detection is demonstrated as an easy method with high precision.

Table 5 Result compared with other algorithms (Average)

\begin{tabular}{ccccc}
\hline Method & $u_{0}$ & $v_{0}$ & $\varphi$ & $\alpha$ \\
\hline Our method & 149.4 & 119.2 & $12.1^{\circ}$ & 369.6 \\
Vanishing point & 154.2 & 120.2 & $11.8^{\circ}$ & 360.1 \\
Corner & 150.3 & 119.4 & $12.0^{\circ}$ & 373.8 \\
6-point method & 149.4 & 120.4 & $12.2^{\circ}$ & 374.2 \\
\hline
\end{tabular}



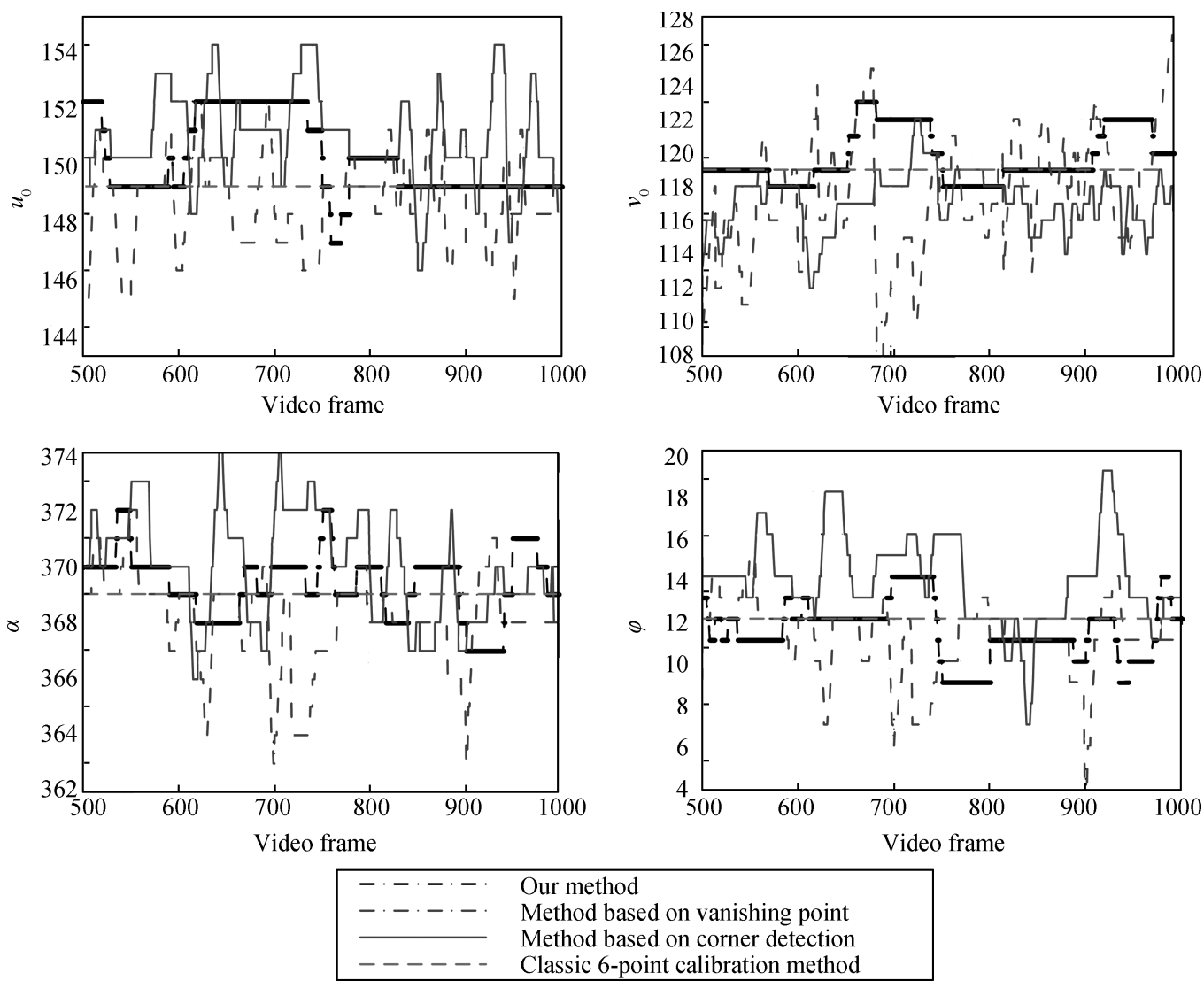

Fig. 16 Calibration compared with three different algorithms

In velocity calculation stage, for each weather condition, we select three frames to illustrate our algorithm. Three vehicles are detected and tracked in sunny weather condition and three for snowy weather condition. Once a vehicle is detected, we use Camshift algorithm to track this vehicle. While in fog weather condition, much more vehicles are detected and tracked. We only choose three vehicles. Thus, data for nine vehicles is collected. We name these nine vehicles by their location in the traffic video scene from left to right in three weather conditions, respectively. In Figs. 17-19, from left to right, we call the vehicles frame by VS.A, VS.B, VS.C, VN.A, VN.B, VN.C, VF.A, VF.B and VF.C, respectively. The real distance and velocity data we used are the scene distance counted by manual. This distance and velocity are taken as standard data. The distance and velocity we calculated by the proposed algorithm will be contrasted with the standard data. We denote F.L to stand for frame left, F.M to stand for frame middle, F.R to stand for frame right, LTM stands for the period of the left frame to the middle frame and MTR stands for the period from the middle frame to the right frame in all of these three weather condition video sequences. From Tables 6-11, "real" stands for standard data measured manually, and "detect" stands for data calculated by our presented algorithm. The ultimate results show that the velocity calculating precision is more than $95 \%$.

According to the relationship between frames and time consumption in processing, we compute the running time between the left frame, the middle frame and the right frame. Consuming time from the left frame to the middle frame in sunny condition (Fig. 17) is $300 \mathrm{~ms}$. Time between the middle frame and the right frame for sunny condition is $580 \mathrm{~ms}$. Then velocity of these three vehicles could be calculated according to (49). Tables 6 and 7 show the distance and velocity calculation results for sunny condition, respectively.
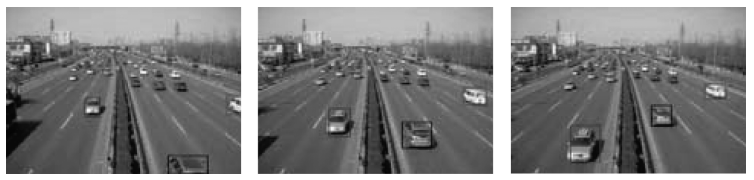

Fig. 17 Sunny vehicle detection and tracking results

Table 6 Distance data for sunny condition

\begin{tabular}{ccccccc}
\hline \multirow{2}{*}{ Vehicle } & \multicolumn{3}{c}{ Real $(\mathrm{m})$} & \multicolumn{3}{c}{ Detect $(\mathrm{m})$} \\
& F.L & F.M & F.R & F.L & F.M & F.R \\
\hline VS.A & 15.8 & 12.8 & 6.7 & 15.4 & 12.5 & 6.3 \\
VS.B & 2 & 8.1 & 14 & 2.1 & 8.3 & 14.3 \\
VS.C & 15.4 & 19.3 & 28.6 & 15.6 & 19.6 & 28.4 \\
\hline
\end{tabular}

Table 7 Velocity data for sunny condition

\begin{tabular}{ccccccc}
\hline Vehicle & $\begin{array}{c}\text { LTM } \\
\text { real } \\
(\mathrm{km} / \mathrm{s})\end{array}$ & $\begin{array}{c}\text { LTM } \\
\text { detect } \\
(\mathrm{km} / \mathrm{s})\end{array}$ & $\begin{array}{c}\text { Right } \\
\text { ratio } \\
(\%)\end{array}$ & $\begin{array}{c}\text { MTR } \\
\text { real } \\
(\mathrm{km} / \mathrm{s})\end{array}$ & $\begin{array}{c}\text { MTR } \\
\text { detect } \\
(\mathrm{km} / \mathrm{s})\end{array}$ & $\begin{array}{c}\text { Right } \\
\text { ratio } \\
(\%)\end{array}$ \\
\hline VS.A & 36 & 34.8 & 95 & 37.8 & 38.4 & 98.4 \\
VS.B & 73.2 & 74.4 & 98.3 & 36.6 & 37.2 & 98.3 \\
VS.C & 46.8 & 48 & 98 & 57.7 & 54.6 & 95 \\
\hline
\end{tabular}


Consuming time from the left frame to the middle frame for snowy condition (Fig. 18) is $1500 \mathrm{~ms}$. Time between the middle frame and the right frame for snowy condition is $1800 \mathrm{~ms}$. Tables 8 and 9 show the distance and velocity results for snow weather condition, respectively. It is much more meaningful to detect vehicle velocity in snowy weather condition. For example, it could tell driver to drive more slowly. Lane in snowy weather condition could not be detected, so many algorithms fail. Our algorithm proposed in this paper gives an effective method.

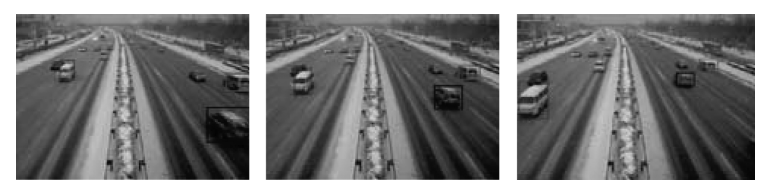

Fig. 18 snowy vehicle detection and tracking results

Time between the left frame and the middle frame for fog (Fig.19) is $1800 \mathrm{~ms}$. Time between the middle frame and the right frame for snowy weather condition is $800 \mathrm{~ms}$. Tables 10 and 11 show the distance and velocity results for snow weather condition, respectively.

Table 8 Distance data for snowy condition

\begin{tabular}{ccccccc}
\hline \multirow{2}{*}{ Vehicle } & \multicolumn{3}{c}{ Real $(\mathrm{m})$} & \multicolumn{3}{c}{ Detect $(\mathrm{m})$} \\
\cline { 2 - 7 } & F.L & F.M & F.R & F.L & F.M & F.R \\
\hline VN.A & 41.2 & 30.4 & 16.1 & 41.4 & 30.1 & 16 \\
VN.B & 7.2 & 19 & 38 & 7.6 & 19.2 & 43.2 \\
VN.C & 15.4 & 38.4 & 43.2 & 15.2 & 38.1 & 43 \\
\hline
\end{tabular}

Table 9 Velocity data for snowy condition

\begin{tabular}{ccccccc}
\hline Vehicle & $\begin{array}{c}\text { LTM } \\
\text { real } \\
(\mathrm{km} / \mathrm{s})\end{array}$ & $\begin{array}{c}\text { LTM } \\
\text { detect } \\
(\mathrm{km} / \mathrm{s})\end{array}$ & $\begin{array}{c}\text { Right } \\
\text { ratio } \\
(\%)\end{array}$ & $\begin{array}{c}\text { MTR } \\
\text { real } \\
(\mathrm{km} / \mathrm{s})\end{array}$ & $\begin{array}{c}\text { MTR } \\
\text { detect } \\
(\mathrm{km} / \mathrm{s})\end{array}$ & $\begin{array}{c}\text { Right } \\
\text { ratio } \\
(\%)\end{array}$ \\
\hline VN.A & 25.92 & 27.2 & 95 & 28.6 & 28.2 & 98.6 \\
VN.B & 28.3 & 27.8 & 98 & 45.6 & 48 & 95 \\
VN.C & 55.2 & 54.9 & 99.4 & 9.6 & 9.8 & 95 \\
\hline
\end{tabular}
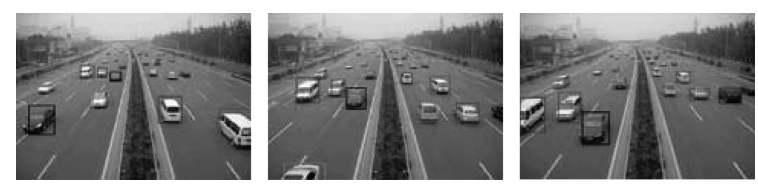

Fig. 19 Vehicle detection and tracking results

Table 10 Distance data for fog

\begin{tabular}{ccccccc}
\hline \multirow{2}{*}{ Vehicle } & \multicolumn{3}{c}{ Real $(\mathrm{m})$} & \multicolumn{3}{c}{ Detect $(\mathrm{m})$} \\
\cline { 2 - 7 } & F.L & F.M & F.R & F.L & F.M & F.R \\
\hline VF.A & 46.4 & 21 & 16 & 46.1 & 21.2 & 16.3 \\
VF.B & 41 & 18 & 10 & 40.7 & 19.1 & 11 \\
VF.C & 8 & 28 & 39.4 & 8.1 & 28.2 & 39 \\
\hline
\end{tabular}

Table 11 Velocity data for fog condition

\begin{tabular}{ccccccc}
\hline Vehicle & $\begin{array}{c}\text { LTM } \\
\text { real } \\
(\mathrm{km} / \mathrm{s})\end{array}$ & $\begin{array}{c}\text { LTM } \\
\text { detect } \\
(\mathrm{km} / \mathrm{s})\end{array}$ & $\begin{array}{c}\text { Right } \\
\text { ratio } \\
(\%)\end{array}$ & $\begin{array}{c}\text { MTR } \\
\text { real } \\
(\mathrm{km} / \mathrm{s})\end{array}$ & $\begin{array}{c}\text { MTR } \\
\text { detect } \\
(\mathrm{km} / \mathrm{s})\end{array}$ & $\begin{array}{c}\text { Right } \\
\text { ratio } \\
(\%)\end{array}$ \\
\hline VS.A & 50 & 49 & 98 & 22.5 & 22.1 & 98 \\
VS.B & 44 & 43.2 & 98 & 36 & 35.8 & 99 \\
VS.C & 40 & 40.2 & 99 & 51.3 & 48.6 & 95 \\
\hline
\end{tabular}

All of the algorithms are run on a PC with $1.0 \mathrm{GHz}$ Intel Pentium 4 processor. These three traffic video sequences are sized by $320 \times 240$. The average running time for these three main stages (weather condition recognition, camera parameter calibration, velocity calculation) is about $10 \mathrm{~ms}$, $20 \mathrm{~ms}$ and $10 \mathrm{~ms}$ respectively to process an image. The total time consumed is $40 \mathrm{~ms}$. This running time could maintain real time processing.

\section{Conclusions}

In this paper, a novel algorithm for vehicle average velocity calculation through automatic and dynamic camera calibration based on dark channel prior in homogenous fog weather condition is presented. Camera fixed in the middle of the road should be calibrated in homogenous fog weather condition, and can be used in any weather condition. Unlike other researches in velocity detection area, in our traffic model, only road plane and vehicles in motion are included. Painted lines in scene image are neglected because sometimes there are no traffic lanes, especially in un-structured traffic scene. Once calibrated, distance information will be got and can be used to calculate vehicles average velocity. At the end of this paper, calibration results and vehicles velocity data for nine vehicles in different weather conditions are given. Comparison with three other algorithms verifies the effectiveness of our algorithm. In the future research, we plan to achieve camera calibration in different tilt angle, especially roadside cameras. Nonlinear camera distortion model will be introduced into the foggy traffic model which will make the calibration results more consistent with the actual situation. Research results in this paper provide a new method on vehicle velocity detection under different weather conditions and contribute to the fog visibility detection and traffic scene $3-\mathrm{D}$ reconstruction.

\section{References}

[1] G. Toulminet, M. Bertozzi, S. Mousset, A. Bensrhair, A. Broggi. Vehicle detection by means of stereo vision-based obstacles features extraction and monocular pattern analysis. IEEE Transactions on Image Processing, vol. 15, no. 8, pp. 2364-2375, 2006.

[2] Y. K. Ki, D. K. Baik. Model for accurate speed measurement using double-loop detectors. IEEE Transactions on Vehicular Technology, vol.55, no. 4, pp. 1094-1101, 2006.

[3] N. Hautiére, J. P. Tarel, J. Lavenant, D. Aubert. Automatic fog detection and estimation of visibility distance through use of an onboard camera. Machine Vision and Applications, vol. 17, no. 1, pp.8-20, 2006.

[4] H. Y. Lin, K. J. Li. Motion blur removal and its application to vehicle speed detection. In Processings of IEEE In- 
ternational Conference on Image, IEEE, Piscataway, USA, pp. 3407-3410, 2004.

[5] D. J. Dailey, F. W. Cathey, S. Pumrin. An algorithm to estimate mean traffic speed using uncalibrated cameras. IEEE Transactions on Intelligent Transportation Systems, vol. 1, no. 2, pp. 98-107, 2000.

[6] Y. I. Adbel, H. M. Karara. Direct linear transformation into object space coordinates in close-range photogrammerty. In Proceedings of the Symposium on Close-range Photogrammetry, Urbana, IL, Venezuela, pp. 1-18, 1971.

[7] Y. T. Li, F. H. Zhu, Y. F. Ai, F. Y. Wang. On automatic and dynamic camera calibration based on traffic visual surveillance. In Proceedings of IEEE Intelligent Vehicles Symposium, IEEE, Istanbul, Turkey, pp. 358-363, 2007.

[8] S. Bouzar, F. Lenoir, J. M. Blosseville, R. Glachet. Traffic measurement: Image processing using road markings. In Proceedings of the 8th International Conference on Road Traffic Monitoring and Control, IEE, London, UK, no. 422, pp. 105-109, 1996.

[9] A. Fusiello. Uncalibrated euclidean reconstruction: a review. Image and Vision Computing, vol. 18, no. 6-7, pp. 555-563, 2000

[10] T. N. Schoepflin, D. J. Dailey. Dynamic camera calibration of roadside traffic management cameras for vehicle speed estimation. IEEE Transactions on Intelligent Transportation Systems, vol. 4, no. 2, pp. 90-98, 2003.

[11] K. M. He, J. Sun, X. O. Tang. Single image haze removal using dark channel prior. In Proceedings of 2009 IEEE Computer Society Conference on Computer Vision and Pattern Recognition, IEEE, Miami, USA, pp. 1956-1963, 2009.

[12] W. E. L. Grimson, C. Stauffer, R. Romano, L. Lee. Using adaptive tracking to classify and monitor activities in a site. In Proceedings of the IEEE Computer Society Conference on Computer Vision and Pattern Recognition, IEEE, Santa Barbara, USA, pp. 22-29, 1998.

[13] B. D. Stewart, I. Reading, M. S. Thomson, T. D. Binnie, K. W. Dickinson, C. L. Wan. Adaptive lane finding in road traffic image analysis. In Proceedings of the 7th International Conference on Road Traffic Monitoring and Control, IEE, London, UK, pp. 133-136, 1994.

[14] F. Guo, A. M. Chen, B. Li. An algorithm to estimate real-time traffic speed using uncalibrated cameras. Journal of Computer-Aided Design \& Computer Graphics, vol. 18, no. 9, pp. 1337-1344, 2006. (in Chinese)
[15] B. Li, R. Dong, Q. M. Chen. Automatic calibration method for PTZ camera. Journal of Beijing University of Posts and Telecommunications, vol. 32, no. S1, pp. 25-29, 2009. (in Chinese)

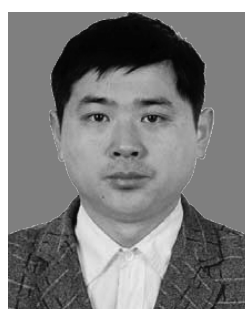

Hong-Jun Song graduated from Qufu Normal University, China in 2005. He received the B. Sc. degree from Qufu Normal University in 2005 , and the M. Sc. degree in mathematics and applied mathematics and cybernetics from Qufu Normal University, China in 2008. He is currently a Ph. D. candidate in pattern recognition and intelligent system at College of Electronic information and Control Engineering, Beijing University of Technology, China.

His research interests include computer vision, intelligent transportation system, and image processing.

E-mail: songhongjun2001@yahoo.cn

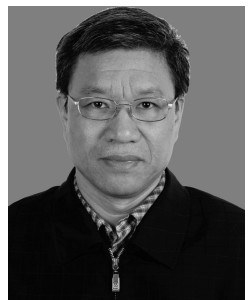

Yang-Zhou Chen received his $\mathrm{Ph} . \mathrm{D}$. degree in cybernetics in 1994 from St. Petersburg University, the Russian Federation. From 1996 to 1998, he was a postdoctoral fellow in College of Astronautics at Harbin Institute of Technology, China. From 2005 to 2006, he worked as a visiting professor at Electrical and Computer Engineering Department, University of Toronto, Canada. He is a professor and currently working in Institute of Autonomous Technology and Intelligent Control, Beijing University of Technology, China.

His research interests include hybrid dynamic systems with discrete event dynamic systems, intelligent transportation systems, and multi-vehicle (mobile robot) system of cooperative control.

E-mail: yzchen@bjut.edu.cn

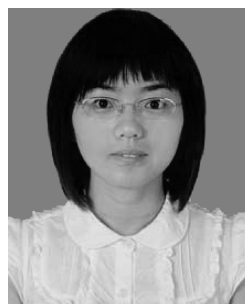

Yuan-Yuan Gao received her B. Sc. degree in biomedical engineering from College of Electrical Engineering, Zhengzhou University, China in 2007. She received the $\mathrm{Ph} . \mathrm{D}$. degree in pattern recognition and intelligent system at College of Electronic information and Control Engineering Beijing University of Technology, China in 2012. She is currently working in the College of Information and Engineering, Zhejiang Agriculture and Forestry University.

Her research interests include robots, automatic control, and path planning.

E-mail: yuangao84@163.com (Corresponding author) 